\title{
Agroindustrial Science
}

Escuela de Ingeniería Agroindustrial

Website: $\underline{\text { http://revistas.unitru.edu.pe/index.php/agroindscience }}$

Universidad Nacional de Trujillo

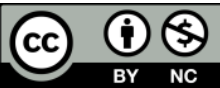

Esta obra está publicada bajo la licencia CC BY-NC 4.0

\section{Enmiendas orgánicas y fitorremediación de cadmio y plomo por lechuga (Lactuca sativa L.) en un suelo agrícola contaminado}

Organic amendments and phytoremediation of $\mathrm{Cd}$ and $\mathrm{Pb}$ by lettuce (Lactuca sativa L.) in a contaminated agricultural soil

\section{Andrés Alberto Azabache Leyton1, *; Juan De La Paz Rodríguez; Betsy Liz Argomedo Vásquez ${ }^{1}$; Ingrid Suail Galván Orcón ${ }^{1}$}

1 Universidad Continental, Escuela de Ingeniería Ambiental, Avenida San Carlos 1980, Huancayo, Perú.

ORCID de los autores

A. A. Azabache Leyton: https://orcid.org/0000-0003-1128-3678 J. De La Paz Rodríguez: https://orcid.org/0000-0002-9813-0078

B. L. Argomedo Vásquez: https://orcid.org/0000-0001-5957-3338 $\quad$ I. S. Galván Orcón: https://orcid.org/0000-0002-2616-9504

\section{RESUMEN}

La contaminación del suelo por metales pesados es un problema creciente en todo el mundo, siendo necesario investigar técnicas sostenibles de mitigación. El objetivo fue determinar el efecto de enmiendas orgánicas en la fitorremediación de cadmio (Cd) y plomo (Pb) por Lactuca sativa L. en un suelo agrícola del distrito de Huancaní, provincia de Jauja. Los tratamientos fueron: vermicompost (VC), estiércol de cuy (EC) y biochar (BCH), al $10 \%$ y $20 \%$, más un testigo, dispuestos en un diseño completamente al azar. Se evaluó $\mathrm{Cd}$ y $\mathrm{Pb}$ en raíz, hojas y suelo, calculando los factores de bioconcentración (FBC), translocación (FT) y remediación (FR). Los contenidos iniciales de $\mathrm{Cd}$ y Pb en el suelo superaron el Estándar de Calidad Ambiental (ECA). El BCH (20\%) incrementó Cd en hojas y raíces, disminuyó Pb en raíz e incrementó $\mathrm{Pb}$ en hojas. Las concentraciones de $\mathrm{Pb}$ y $\mathrm{Cd}$ en lechuga superaron los límites permisibles. El FBC de $\mathrm{Cd}$ y $\mathrm{Pb}$ fue $<1$; el FT fue $>1$ para $\mathrm{Cd}$ en algunos tratamientos con enmiendas orgánicas y para $\mathrm{Pb}$ con $\mathrm{BCH}(20 \%)$. Se concluye que Lactuca sativa L., tuvo un $\mathrm{FR}$ de $55,88 \%$ para $\mathrm{Cd}$ y $51,75 \%$ para $\mathrm{Pb}$, considerándose una planta fitoextractora.

Palabras clave: fitorremediación; enmiendas orgánicas; Lactuca sativa L.; contaminación; cadmio; plomo.

\section{ABSTRACT}

Soil contamination by heavy metals is a growing problem around the world, requiring research on sustainable mitigation techniques. The objective was to determine the effect of organic amendments on the phytoremediation of cadmium (Cd) and lead $(\mathrm{Pb})$ by Lactuca sativa $\mathrm{L}$. in an agricultural soil in the district of Huancaní, province of Jauja. The treatments were: vermicompost (VC), guinea pig manure $(\mathrm{EC})$ and biochar $(\mathrm{BCH})$, at $10 \%$ and $20 \%$, plus a control, arranged in a completely random design. $\mathrm{Cd}$ and $\mathrm{Pb}$ were evaluated in roots, leaves and soil, calculating the bioconcentration factors (BCF), translocation (FT) and remediation (FR). The initial contents of $\mathrm{Cd}$ and $\mathrm{Pb}$ in the soil exceeded the Environmental Quality Standard (ECA). The BCH (20\%) increased $\mathrm{Cd}$ in leaves and roots, decreased $\mathrm{Pb}$ in roots and increased $\mathrm{Pb}$ in leaves. The concentrations of $\mathrm{Pb}$ and $\mathrm{Cd}$ in lettuce exceeded the permissible limits. The $\mathrm{BCF}$ of $\mathrm{Cd}$ and $\mathrm{Pb}$ was $<1$; the FT was $>1$ for $\mathrm{Cd}$ in some treatments with organic amendments and for $\mathrm{Pb}$ with $\mathrm{BCH}(20 \%)$. It is concluded that Lactuca sativa $\mathrm{L}$., had a FR of $55.88 \%$ for $\mathrm{Cd}$ and $51.75 \%$ for $\mathrm{Pb}$, considering it a phytoextractor plant.

Keywords: phytoremediation; organic amendments; Lactuca sativa L.; contamination; cadmium; lead. 


\section{Introducción}

La generación, distribución y derrames accidentales de diferentes compuestos orgánicos e inorgánicos, provenientes de herbicidas, insecticidas, hidrocarburos, relaves mineros, residuos sólidos, entre otros, ha causado contaminación y degradación ambiental directa 0 indirectamente acumulada en suelo, agua y aire (Méndez et al., 2018).

Entre los elementos potencialmente tóxicos, el contenido de metales pesados en un suelo, normalmente se debe a la composición del material de origen y a los procesos pedogenéticos que se presentan con diferente intensidad, pero la actividad humana ha influenciado su concentración y movilidad, a través de la generación de residuos sólidos, líquidos y gaseosos, que llegan al suelo en forma directa 0 indirecta (Rajhumar et al., 2009).

El contenido de elementos tóxicos en suelos agrícolas, su bioacumulación en cultivos alimenticios y su dispersión en el ambiente son las principales vías de exposición para los humanos (Chen et al., 2018). Por esta razón, la descontaminación de suelos se hace una necesidad (Cioica et al., 2019).

En el valle del Mantaro, las aguas del río del mismo nombre son utilizadas para el riego agrícola, habiéndose detectado concentraciones de metales pesados, superiores al límite máximo permisible de Canadá; y en la zona media de esta cuenca, se observó distribución homogénea de estos contaminantes. Estos sugieren que la acumulación de metales y metaloides a nivel superficial tienen origen antropogénico pudiendo ser transportados a través del agua en la zona media (Proyecto Mantaro Revive, 2007).

El rápido incremento de la contaminación del suelo con metales pesados en las últimas décadas son una gran amenaza para la salud humana y el ambiente (Muthusaravanan et al., 2018). La remoción de estos contaminantes inorgánicos requiere de tecnologías efectivas y soluciones de bajo costo, pues los metales pesados son contaminantes que no pueden ser descompuestos a formas menos tóxicas (Cameselle \& Gouveia, 2019). Entre estos elementos, el cadmio ( $\mathrm{Cd}$ ), el plomo ( $\mathrm{Pb}$ ), el níquel (Ni) y el mercurio $(\mathrm{Hg})$ se mostraron como los contaminantes más frecuentes en muestras de suelo a través del mundo (Mahar et al., 2016; Oconnor et al., 2019). Debido al incremento de problemas de salud asociados con metales pesados y su amplia distribución en los suelos, se ha tomado énfasis en identificar las fuentes de contaminación y a remediar sitios contaminados (Duan et al., 2017).

El uso de métodos físicos y químicos para descontaminar suelos incluyen el uso de diferentes técnicas, como el intercambio iónico, la ósmosis reversa, la reducción química, la precipitación, la evaporación, el lavado, la electrocinética y tratamientos de calor (Habibul et al., 2019). Todas ellas pueden ser efectivamente aplicadas en la descontaminación del suelo, pero requieren una fuente externa de insumos y en la mayoría de los casos son costosas para un uso a gran escala (Sharma et al., 2015).

Dentro de las tecnologías de remediación de suelos, la fitorremediación, que usa plantas como la principal fuente, se constituye en una alternativa ecológica para descontaminar los suelos de una determinada área, es de bajo costo, efectiva, y puede ser implementada in si tu y ex situ, con la utilización de enmiendas para favorecer su efectividad y mejorar propiedades del suelo (Arnold et al., 2020; Yadav et al., 2018). La aplicación de enmiendas orgánicas o inorgánicas a los suelos contaminados puede reducir la movilidad debido a cambios en las propiedades del suelo (Bernal et al., 2020).

Do Carmo et al. (2019) evaluaron la toxicidad de sedimentos a través de un bioensayo utilizando Lactuca sativa y compost para favorecer la inmovilización de metales pesados. La enmienda redujo la cantidad de metales extractables y mejoró los parámetros morfológicos del vegetal.

FAO (2011) reporta en El Reglamento Técnico Mercosur sobre Límites Máximos Permisibles de Contaminantes Inorgánicos en alimentos que, para hortalizas de hoja, donde se incluye Lactuca

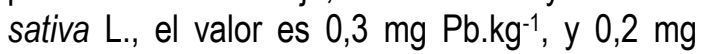
Cd.kg-1. Ruiz (2011) evaluó Lactuca sativa L. para disminuir cadmio ( $\mathrm{Cd}$ inicial $=3,97 \mathrm{mg} \cdot \mathrm{kg}^{-1}$ ) en suelos regados con aguas contaminadas del río Bogotá, y aplicó $\mathrm{CaCO} 3$ y compost antes del transplante, reduciendo el $\mathrm{Cd}$ en el suelo a 3,38 $\mathrm{mg} \cdot \mathrm{kg}^{-1}$.

Madueño (2017) reportó la concentración de cadmio y plomo en Lactuca sativa L. var. Crespa en 20 mercados de Lima, Perú, obteniendo una media de 1,279 y $0,084 \mathrm{ppm}$, respectivamente, superando el nivel máximo para $\mathrm{Pb}$, pero no para Cd.

Gunduz et al. (2012) evaluaron el potencial de fitoremediación de varias especies hortícolas en áreas mineras de Chipre, encontrando que la lechuga, con factor de remediación $12,92 \%$, fue la especie más adecuada para plomo; y el cadmio solo alcanzó 1,43\%, considerado bajo. 
Musa et al. (2017) colectaron muestras de suelo y lechuga en tres parcelas circundantes al río Salanta, Nigeria, encontrando en el suelo: 10 a 20 $\mathrm{mg} \mathrm{Cd} / \mathrm{kg}$, y 6,5 a $21,7 \mathrm{mg} \mathrm{Pb} / \mathrm{kg}$. En Lechuga: 10 - $20 \mathrm{mg} \mathrm{Cd} / \mathrm{kg}$ y $2,1-6,5 \mathrm{mg} \mathrm{Pb} / \mathrm{kg}$. Todos superiores a los límites permisibles de la Organización Mundial de la Salud. El factor de bioconcentración fue 0,7 a 2,0 para Cd y 0,19 a 0,70 para $\mathrm{Pb}$, recomendándose no sembrar lechuga en estos suelos.

Purwaningdyah \& Takarina (2019) reportan los factores de bioconcentración (FBC $=$ (Concentración del metal pesado en hoja)/(concentración en suelo) y translocación ( $\mathrm{FT}=$ Concentración del metal pesado en hoja)/(concentración en raíz).

Audet \&Charest (2007) mencionan que cuando el Ft es mayor de 1, la planta tiene gran capacidad para transportar metales desde las raíces a los tallos, debido al secuestro en vacuolas de hojas y apoplasto, por lo que esta puede usarse con fines de fitoextracción.

El biochar, como un nuevo tipo de material funcionalmente ambiental, muestra gran potencial en el mejoramiento del suelo, remediador de la contaminación y en la utilización de recursos de biomasa residuales, habiéndose convertido en un tema de interés en la ciencia agrícola y ambiental (Wang et al., 2018).

El objetivo de este estudio fue evaluar la fitorremediación de $\mathrm{Cd}$ y $\mathrm{Pb}$, por el cultivo de lechuga (Lactuca sativa L.), con la aplicación de enmiendas orgánicas, para descontaminar un suelo de Huancaní, provincia de Jauja, Perú.

\section{Material y métodos}

\section{Zona de estudio}

La investigación utilizó un suelo agrícola del distrito de Huancaní, provincia de Jauja, región Junín. La parcela se ubicó a una altitud de 3328 msnsm, con las coordenadas UTM $454907.36 \mathrm{~m}$ E - $8688430.38 \mathrm{~m} \mathrm{~S}$. La temperatura promedio anual es de $12,5^{\circ} \mathrm{C}$ y precipitación promedio anual de $601 \mathrm{~mm}$ (SENAMHI, 2021).

\section{Planta fitorremediadora}

Lechuga (Lactuca sativa L.) variedad Seda.

\section{Características del suelo}

En el mes de mayo, del año 2021, se seleccionó una parcela agrícola del distrito de Huancani, ubicado en la margen derecha del río Mantaro, que es regada con aguas del río del mismo nombre, fisiográficamente en terraza baja, que estuvo cultivado con maíz, muestreando la capa superficial en un promedio de profundidad de $0 \mathrm{a}$ $20 \mathrm{~cm}$, mediante la técnica de cuadrícula (Tabla 1).

\section{Tabla 1}

Resultados del análisis de caracterización del suelo agrícola. Centro Poblado Huancani. Distrito de Leonor Ordoñez, Jauja, Junín

\begin{tabular}{|c|c|}
\hline Variable & Resultado \\
\hline $\mathrm{pH}$ & 7,25 \\
\hline Conductividad Eléctrica (dS/m) & 0,84 \\
\hline $\mathrm{CaCO}_{3}\left(\mathrm{~g} \cdot \mathrm{kg}^{-1}\right)$ & 75,00 \\
\hline Materia Orgánica (g.kg-1) & 19,50 \\
\hline Fósforo disponible $\left(\mathrm{mg} \mathrm{kg}^{-1}\right)$ & 17,20 \\
\hline Potasio disponible (mg. $\mathrm{kg}^{-1}$ ) & 209,00 \\
\hline \multicolumn{2}{|l|}{ Textura: } \\
\hline Arena $\left(\mathrm{g} \cdot \mathrm{kg}^{-1}\right)$ & 300 \\
\hline $\operatorname{Limo}\left(\mathrm{g} \cdot \mathrm{kg}^{-1}\right)$ & 410 \\
\hline Arcilla $\left(\mathrm{g} \cdot \mathrm{kg}^{-1}\right)$ & 290 \\
\hline Clase Textural & Franco Arcilloso \\
\hline Capacidad de Intercambio & 1440 \\
\hline Catiónico (cmol. $\left.\mathrm{kg}^{-1}\right)$ & 14,40 \\
\hline \multicolumn{2}{|l|}{ Cationes cambiables (cmol. $\left.\mathrm{kg}^{-1}\right)$ : } \\
\hline $\mathrm{Ca}^{+2}$ & 10,29 \\
\hline $\mathrm{Mg}^{+2}$ & 3,23 \\
\hline $\mathrm{K}^{+}$ & 0,51 \\
\hline $\mathrm{Na}^{+}$ & 0,37 \\
\hline Saturación de Bases (\%) & 100,00 \\
\hline Plomo (mg.kg-1) & 238,04 \\
\hline Cadmio (mg.kg-1) & 8,75 \\
\hline
\end{tabular}

El suelo presentó un pH ligeramente alcalino (7,1 - 7,8), la conductividad eléctrica calificó al suelo como no salino $\left(<0,2 \mathrm{dS} \mathrm{m}^{-1}\right)$, el contenido de calcáreo fue alto $\left(50-150 \mathrm{~g} \mathrm{~kg}^{-1}\right)$, debido al material parental calcáreo, con un contenido bajo de materia orgánica $\left(<20 \mathrm{~g} \mathrm{~kg}^{-1}\right)$; alto contenido de fósforo (> $14 \mathrm{mg} \mathrm{kg}^{-1}$ ), contenido medio de potasio $\left(100-240 \mathrm{mg} \mathrm{kg}^{-1}\right)$, de textura media, que facilitó el desarrollo de la planta. Capacidad de intercambio catiónico moderadamente alta (12 $20 \mathrm{cmol} \mathrm{kg}^{-1}$ ) y una saturación de bases alta (> 50 $\%$ ). Los contenidos de $\mathrm{Pb}$ y $\mathrm{Cd}$ superaron el ECA de suelos del Perú, presentando con un factor de contaminación ( $\mathrm{FC}=$ concentración en muestral concentración $\mathrm{ECA}$ ) de 2,97 y 2,25 para $\mathrm{Pb}$ y $\mathrm{Cd}$, respectivamente (MINAM, 2017).

\section{Enmiendas orgánicas}

El biochar, obtenido del estiércol de ovino, tuvo $\mathrm{pH}$ alcalino, con mínima concentración de sales. Contenido de nitrógeno, fósforo, potasio y materia orgánica, dentro de los parámetros de una enmienda orgánica, según la Norma Técnica Colombiana (NTC, 2011). El vermicompost tuvo exceso de sales y pH ácido. Los demás datos 
estuvieron dentro de los rangos normales, reportando mayor contenido de $\mathrm{N}$ total y $\mathrm{K}_{2} \mathrm{O}$ que el biochar. Los datos se presentan en la tabla 2. Estiércol de cuy descompuesto, se utilizó de un criadero familiar. Cada enmienda orgánica se aplicó en dos dosis: $10 \%$ y $20 \%$ y se tuvo un tratamiento control sin enmienda. Los datos se presentan en la tabla 2.

Tabla 2

Resultados del análisis del biochar y vermicompost

\begin{tabular}{lcc}
\hline \multirow{2}{*}{ Análisis } & \multicolumn{2}{c}{ Contenido } \\
\cline { 2 - 3 } & Biochar $^{1}$ & Vermicompost $^{2}$ \\
\hline $\mathrm{pH}$ & 7,57 & 3,96 \\
Conductividad & 1,16 & 6,73 \\
Eléctrica $\left(\mathrm{dS} . \mathrm{m}^{-1}\right)$ & 0,74 & 1,44 \\
Nitrógeno total $(\%)$ & 0,43 & 0,34 \\
$\mathrm{P}_{2} \mathrm{O}_{5}(\%)$ & 0,56 & 1,77 \\
$\mathrm{~K}_{2} \mathrm{O}(\%)$ & 33,03 & 30,65 \\
Materia orgánica $(\%)$ &
\end{tabular}

Fuente: ${ }^{1}$ Bioenergy Ingenieros S.A., Mazamari, Junín. ${ }^{2}$ LASPAF, UNALM, Lima, Perú.

\section{Método de investigación}

El método de investigación fue hipotético deductivo, de tipo aplicada. El experimento tuvo siete tratamientos (T1: control, T2: $10 \%$ vermicompost, T3: $20 \%$ vermicompost, T4: $10 \%$ estiércol de cuy, T5: $20 \%$ estiércol de cuy, T6: 10\% biochar y T7: 20\% biochar). Se trabajó con tres repeticiones, y un total de 21 unidades experimentales, distribuidos en un Diseño Completamente al Azar (DCA).

Se utilizaron 6 plántulas de lechuga variedad "Seda", provenientes de almácigo, que fueron trasplantadas en macetas $4 \mathrm{~kg}$ de substrato, codificadas según los tratamientos. Después de su establecimiento se dejó solo 3 plantas por maceta, con la finalidad de asegurar un buen crecimiento. Se realizaron las labores culturales necesarias de deshierbo, y control fitosanitario en forma manual; el riego se hizo con una frecuencia de 4 días en promedio, utilizando agua potable, manteniendo la humedad al $80 \%$ de la capacidad de campo. Al cabo de 60 días se cosechó el experimento, cortando la parte aérea y la parte radicular para la determinación de materia seca y contenido de $\mathrm{Cd}$ y $\mathrm{Pb}$. Posteriormente se removió y mezcló el suelo de cada maceta para obtener una muestra y enviar al laboratorio para los análisis respectivos.

Cálculo de los indicadores de fitorremediación Los resultados de $\mathrm{Cd}$ y $\mathrm{Pb}\left(\mathrm{mg} \mathrm{kg}^{-1}\right)$ en cada unidad experimental, del suelo, hojas y raíz de lechuga, fueron sistematizados y se calculó el factor de bioconcentración (FBC) de $\mathrm{Cd}(\mathrm{Cd}$ planta/ $\mathrm{Cd}$ suelo); factor de translocación ( $\mathrm{FT}$ ) $(\mathrm{Cd}$ hoja / Cd raíz) (Purwaningdyah \& Takarina, 2019), y factor de remediación (FBC * 100) (Gunduz et al., 2012). Se hicieron similares cálculos de FBC, FT y FR para $\mathrm{Pb}$.

\section{Análisis estadístico}

Los datos evaluados fueron verificados en su distribución normal, utilizando la prueba de Shapiro-Wilks, $\alpha=0,05$. Si tenían distribución normal se aplicó el análisis de variancia y prueba de Duncan, de lo contrario se aplicó la prueba no paramétrica de Kruskall-Wallis. Se utilizó el software INFOSTAT (Di Renzo et al., 2020).

\section{Resultados y discusión}

\section{Concentración de $\mathrm{Cd}$ en hojas y raíces}

La concentración de Cd en la raíz y hojas de lechuga fue significativamente superior en el tratamiento con $20 \% \mathrm{BCH}$, respecto a los demás tratamientos. Estos resultados evidencian una mayor absorción de $\mathrm{Cd}$ por efecto de las enmiendas orgánicas; lo cual se debe a que las enmiendas orgánicas favorecen la efectividad de la fitorremediación al mejorar las propiedades del suelo (Armold et al., 2020; Yadav et al., 2018). Los valores encontrados de $\mathrm{Cd}$ en la parte aérea y raíz de lechuga superaron los Límites Permisibles del Reglamento Mercosur (FAO, 2011), que establece un valor de $0,2 \mathrm{mg} \mathrm{Cd} \mathrm{kg}^{-1}$. Estos datos revelan la necesidad de reducir la extracción de $\mathrm{Cd}$ por lechuga o recomendar no sembrarla en estos suelos, como lo propuso Musa et al. (2017), quienes reportaron 10 a $20 \mathrm{mg} \mathrm{Cd} \mathrm{kg}^{-1}$ en lechuga. Los datos se presentan en la Figura 1.

\section{Concentración de $\mathrm{Pb}$ en hojas y raíces}

La concentración de $\mathrm{Pb}$ en la raíz de lechuga fue significativamente mayor en el tratamiento testigo (T1), y menor en el tratamiento con $20 \% \mathrm{BCH}$. Esto se puede atribuir al efecto de los materiales orgánicos en reducir la movilidad de $\mathrm{Pb}$ en el suelo, debido a una mayor adsorción a la matrix del suelo, como lo reportan Bernal et al. (2020) y Do Carmo et al. (2019). En las hojas el tratamiento con $20 \% \mathrm{BCH}$ superó significativamente a los demás tratamientos, evidenciando la traslocación de $\mathrm{Pb}$ de la raíz a las hojas. Todos los valores superan el límite permisible establecido por el Reglamento técnico Mercosur (FAO, 2011) que lo establece en $0,3 \mathrm{mg} \mathrm{Pb} \mathrm{kg}^{-1}$ para hortalizas de hoja. 


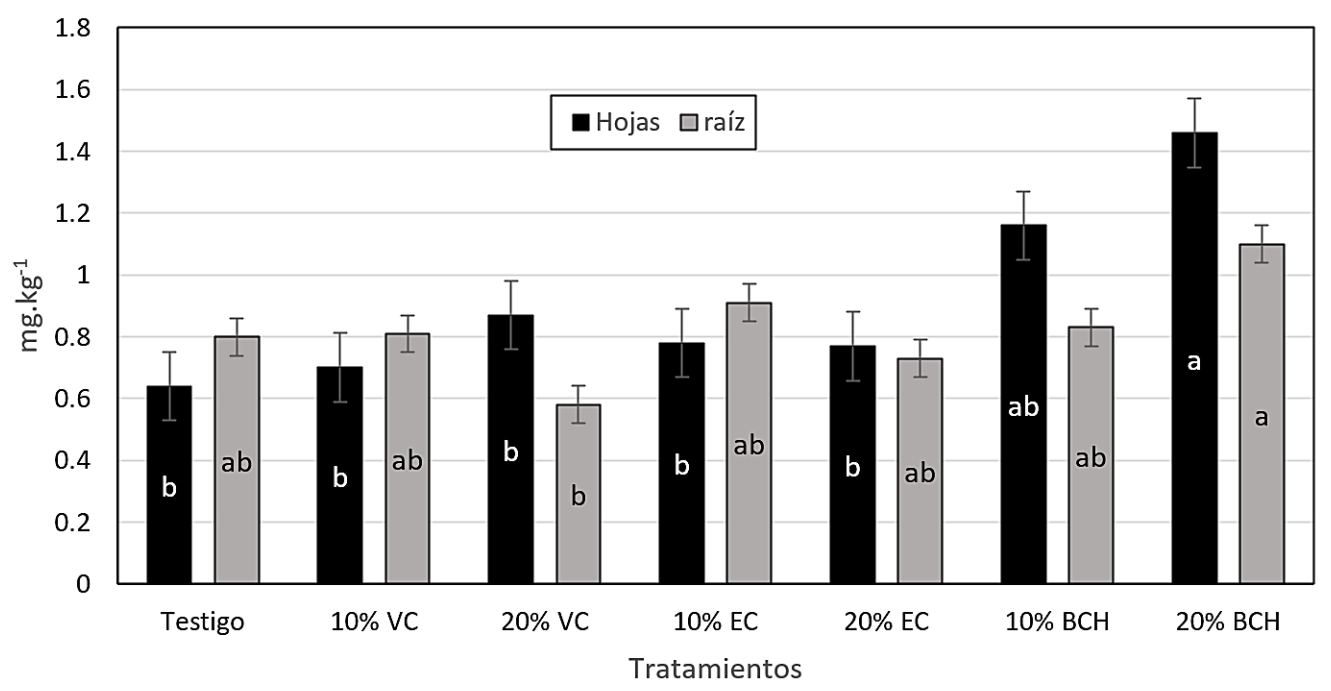

Figura 1. Concentración de $\mathrm{Cd}$ en hojas y raíz de lechuga.

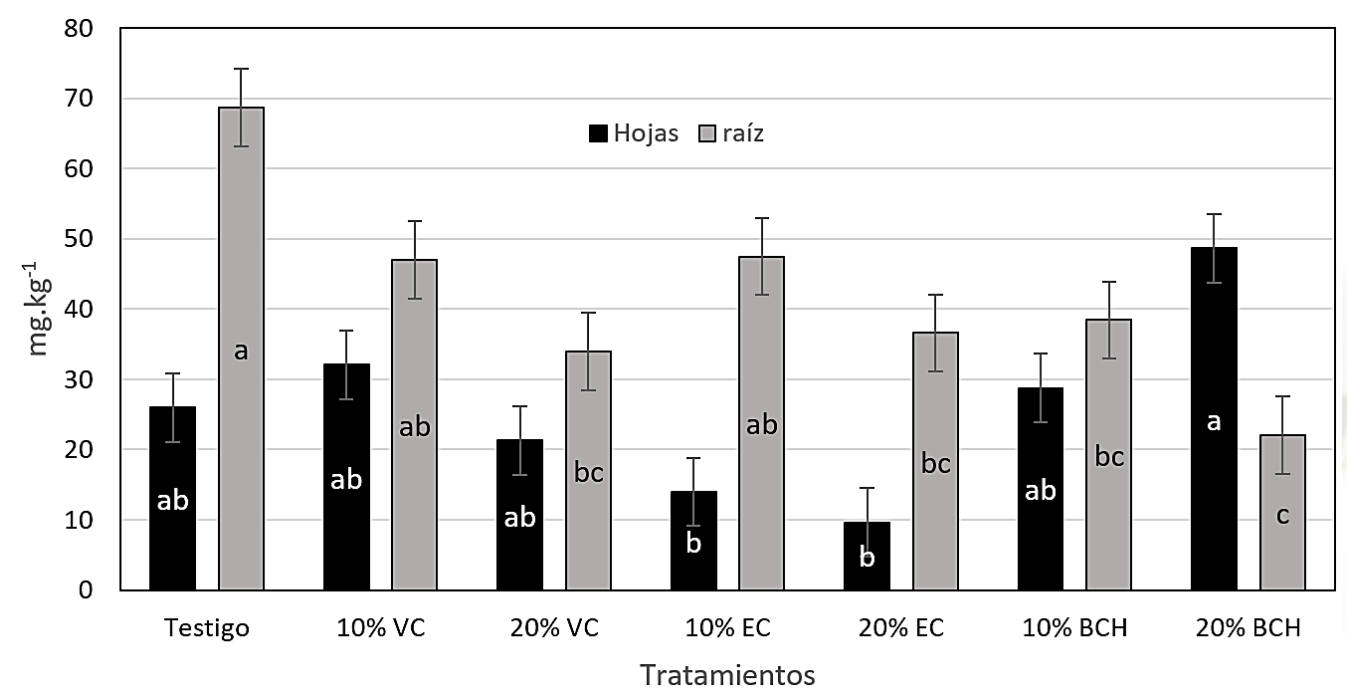

Figura 2. Concentración de Pb en hojas y raíz de lechuga.

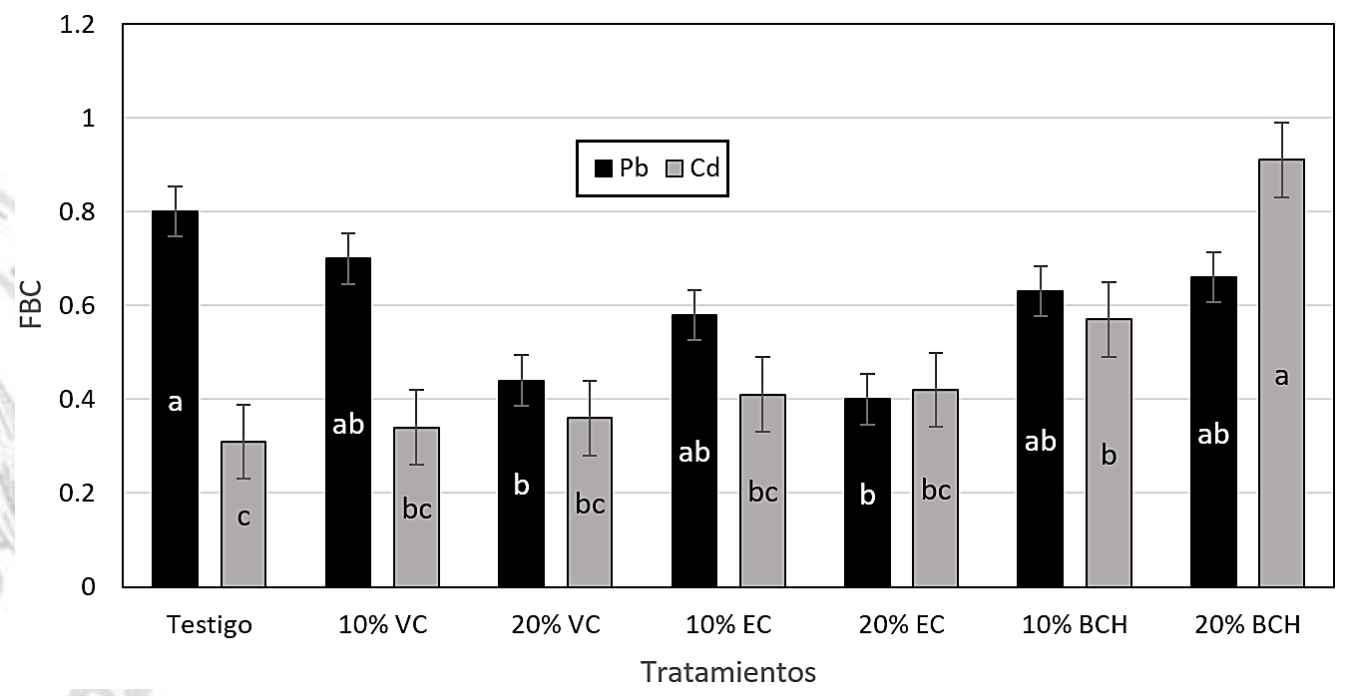

Figura 3. Factores de bioaconcentración de $\mathrm{Cd}$ y Pb. 
Esta alta extracción de $\mathrm{Pb}$ por la lechuga revela la necesidad de no recomendar sembrar esta hortaliza en el suelo de Huancaní, debido al riesgo para la salud humana, como lo propuso Musa et al. (2017), debido a que encontró valores de 2,1 a $6,5 \mathrm{mg} \mathrm{Pb} \mathrm{kg}^{-1}$. Los datos se presentan en la Figura 2.

\section{Efecto de las enmiendas orgánicas en el FBC} Los factores de bioacumulación (FBC) de $\mathrm{Cd}$ y $\mathrm{Pb}$, fueron menores que la unidad indicando que Lactuca sativa $L$. es una planta exclusora 0 estabilizadora de $\mathrm{Pb}$ y $\mathrm{Cd}$. Los resultados de $\mathrm{Cd}$ fueron inferiores a los reportado por Musa et al., (2017), que reportó un $\mathrm{FBC}>1$; lo cual se debe a que tuvo mayores concentraciones de $\mathrm{Cd}$ en el suelo, respecto al suelo del presente experimento. Esto también evidencia el efecto de las enmiendas orgánicas en reducir la movilidad del $\mathrm{Cd}$ y $\mathrm{Pb}$ en el suelo, y su concentración en Lactuca sativa L., como lo reporta Bernal et al. (2020). Los datos se presentan en la Figura 3.

\section{Efecto de las enmiendas orgánicas en el FT}

$\mathrm{El}$ factor de translocación para $\mathrm{Pb}$ fue significativamente mayor que la unidad en el tratamiento con $20 \% \mathrm{BCH}$, mostrando que el $\mathrm{Pb}$ absorbido por las raíces de lechuga es transportado a la parte aérea, cuando se agrega al suelo esta enmienda orgánica. Para el Cd el FT fue superior a la unidad en los tratamientos con $10 \%$ y $20 \% \mathrm{BCH}, 20 \%$ VC y $20 \%$, pero sin diferencias significativas entre todos los tratamientos. Esto evidencia una mayor translocación de $\mathrm{Cd}$ y $\mathrm{Pb}$ con los tratamientos de $\mathrm{BCH}$ indicando que Lactuca sativa $\mathrm{L}$. puede usarse como fioextractora para $\mathrm{Cd} y$ eventualmente para $\mathrm{Pb}$ si se agrega enmiendas orgánicas al suelo contaminado. En este sentido, el biochar es una enmienda orgánica que mejora los suelos contaminados (Wang et al., 2018). Esto está sustentado por Audet \& Charest (2007), quienes sostiene que cuando las plantas tienen un FT mayor que 1, tienen la capacidad de secuestrar el metal pesado en los estomas de las hojas y en el floema. Los datos se presentan en la Figura 4.

\section{Contenido final de $\mathrm{Cd}$ en el suelo}

El contenido final de $\mathrm{Cd}$ en el suelo fue influenciado significativamente por las enmiendas orgánicas, con un promedio de $3,86 \pm 0,36 \mathrm{mg} \mathrm{kg}^{-1}$. El factor de remediación (FR) (Gunduz et al., 2012) de Lactuca sativa L. fue de $55,88 \%$. El tratamiento con $20 \%$ de BCH tuvo menor cantidad de $\mathrm{Cd}$ en el suelo (mayor remoción), debido posiblemente a que el $\mathrm{BCH}$ favorece las propiedades del suelo $y$ mejora la remoción de este metal pesado, como lo reportaron Arnold et al. (2020) y Yadav et al., (2018). Los datos se reportan en la Figura 5.

\section{Contenido final de $\mathrm{Pb}$ en el suelo}

El contenido de $\mathrm{Pb}$ en el suelo disminuyó en todos los tratamientos, con un promedio de 114,82 \pm $17,25 \mathrm{mg} \mathrm{kg}^{-1}$, pero sin diferencias significativas entre los tratamientos. El FR fue de 51,75\%, mayor que lo reportado por Gunduz et al. (2012) para este metal pesado, pues solo reportaron $12,92 \%$, indicando que las propiedades del suelo y la variedad influyen en la fitorremediación. Los datos se presentan en la Figura 6 .

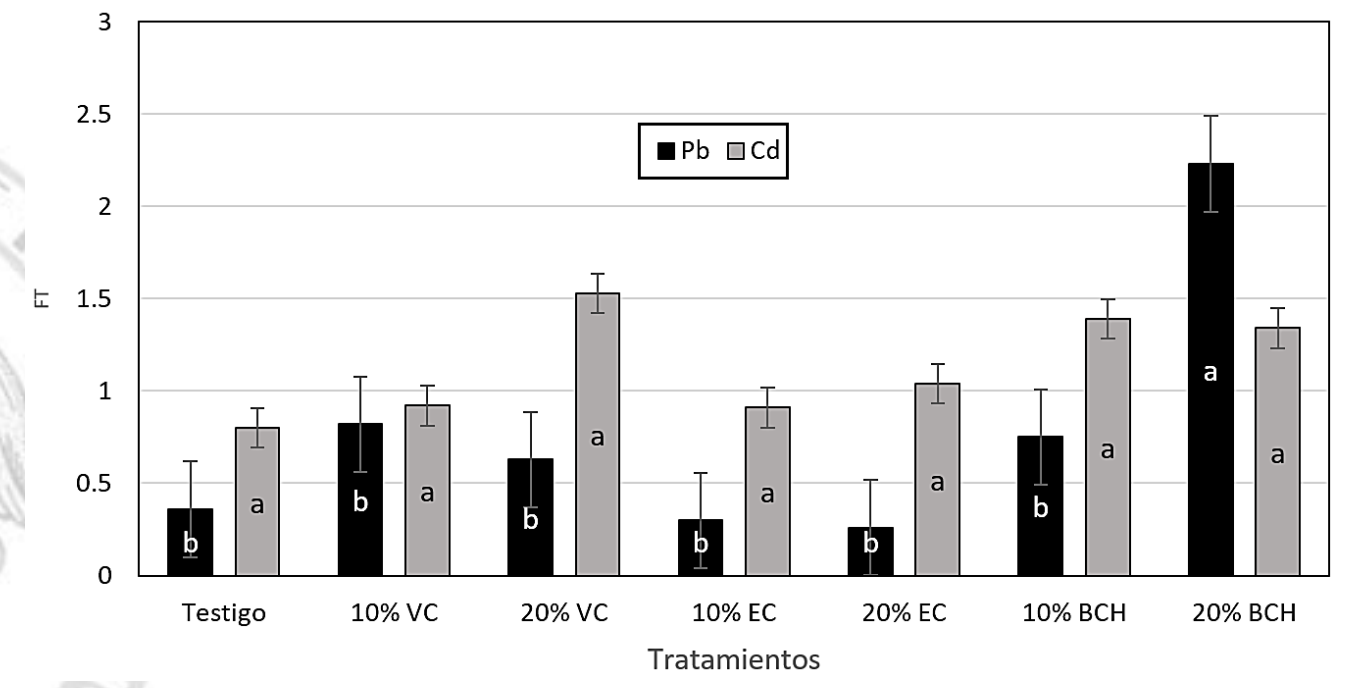

Figura 4. Factor de translocación de $\mathrm{Cd}$ y $\mathrm{Pb}$ en lechuga. 


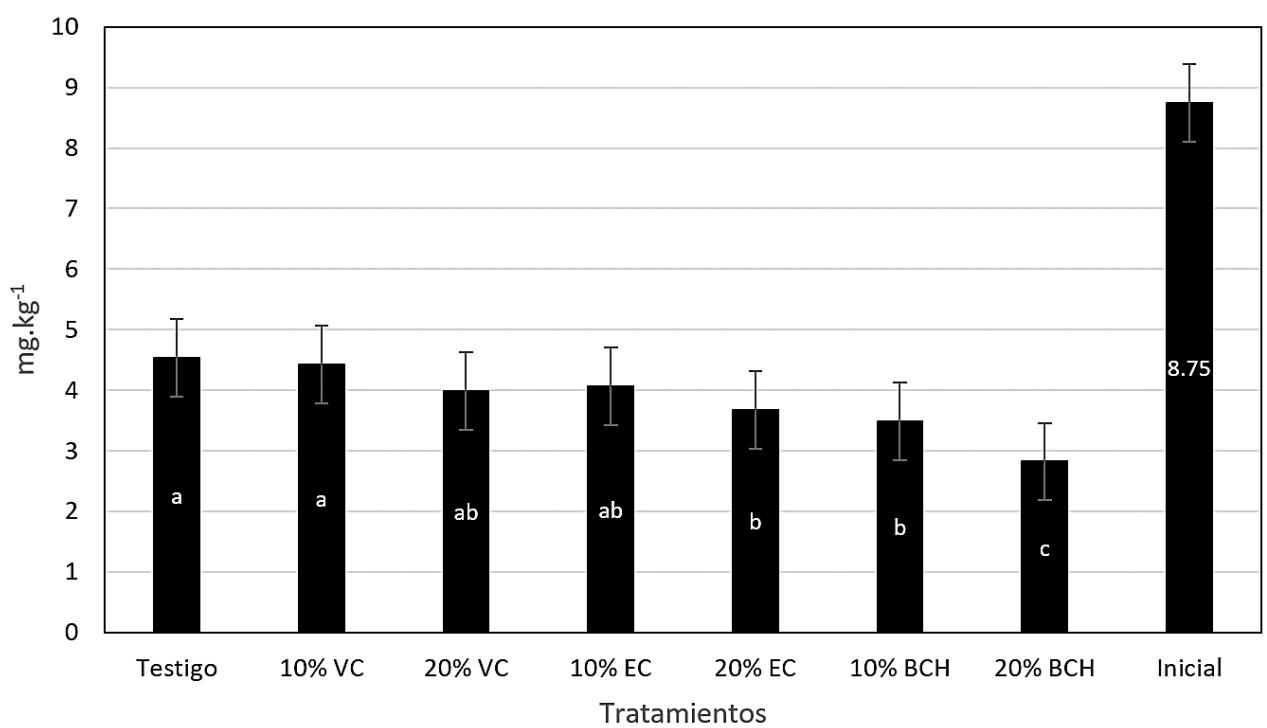

Figura 5. Contenido de Cd en el suelo, después de la fitorremediación.

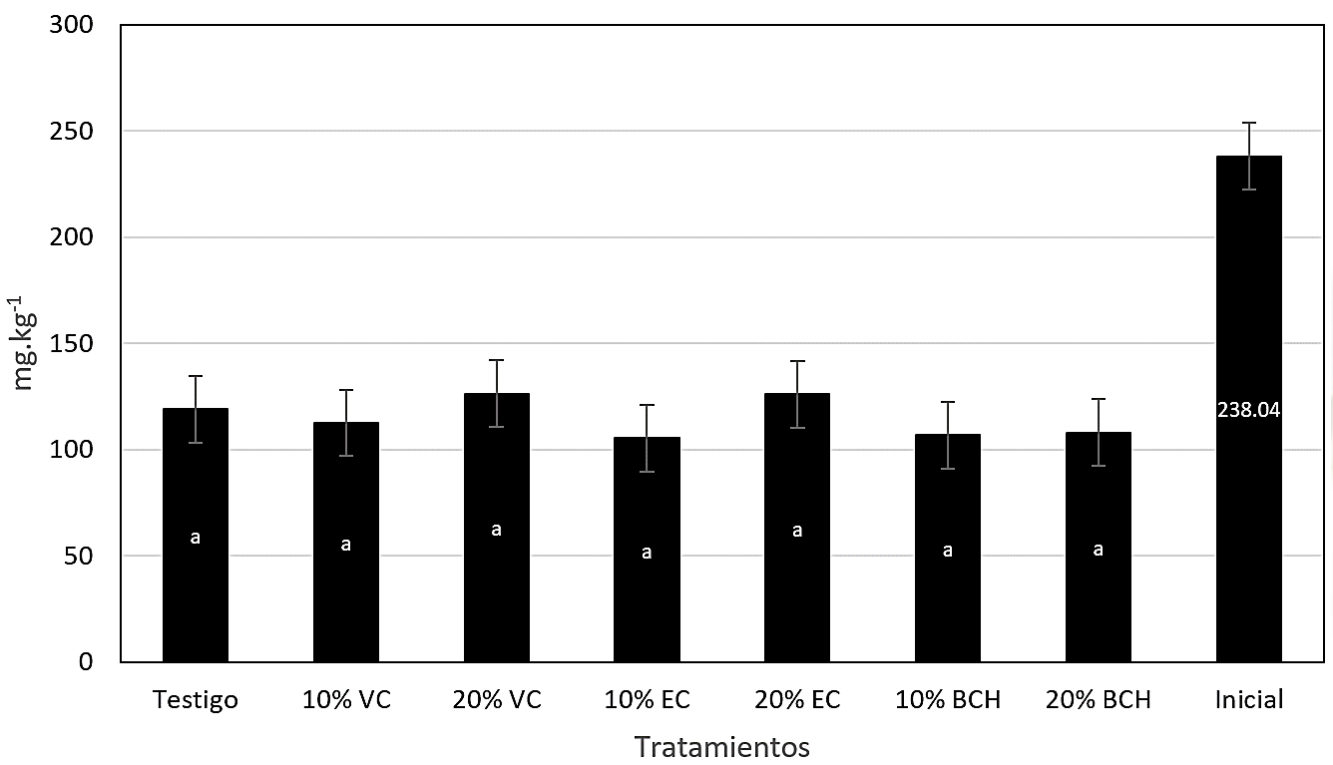

Figura 6. Contenido de $\mathrm{Pb}$ en el suelo, después de la fitorremediación.

\section{Conclusiones}

El biochar, enmienda orgánica obtenida del estiércol de ovino, incrementó significativamente la concentración de $\mathrm{Cd}$ en las hojas y raíz de lechuga. El biochar disminuyó significativamente la concentración de $\mathrm{Pb}$ en la raíz de lechuga, y lo incrementó en las hojas. La biococentración de $\mathrm{Cd}$ y $\mathrm{Pb}$ fue inferior a la unidad, indicando que la lechuga es una planta estabilizadora de estos dos metales pesados. El factor de translocación fue mayor que la unidad para cadmio en los tratamientos con $20 \%$ biochar, $10 \%$ biochar, $20 \%$ VC y $20 \%$ estiércol de cuy, indicando que Lactuca sativa L. puede utilizarse como una planta fitoextractora. Para $\mathrm{Pb}$ la translocación fue significativamente superior en el tratamiento con $20 \%$ biochar. La remediación fue de $55,88 \%$ para
$\mathrm{Cd}$ y de $51,75 \%$ para $\mathrm{Pb}$. Se recomienda restringir la siembra de lechuga en los suelos de Huancani, debido a que concentran el $\mathrm{Cd}$ y $\mathrm{Pb}$ en hojas y raíces, superior a los límites permisibles, e incrementa el riesgo de contaminación en humanos y animales a través de la cadena alimenticia.

\section{Referencias bibliográficas}

Arnold, G., Vladut, N., Voicea, I., Vanghele, N., \& Pruteanu M. (2020). Removal of heavy metals from a contaminated soil using phytoremediation. MATEC Web of Conferences, 305, 00061.

Audet, P., \& Charest, C. (2007). Heavy metal phytoremediation from a meta-analytical perspective. Environmental Pollution, 147(1), 231-237.

Bernal, M., Alvarenga, P., Carmody, K., Pogrzeba, M., \& Soja, G. (2020). Biological remediation of contaminated agricultura soils. EIP-AGRI, Minipaper 3, 2-19. 
Cameselle, C., \& Gouveia, S. (2019). Phytoremediation of mixed contaminated soil enhanced with electric current. J. Hazard. Mater., 361, 95-102.

Chen L., Zhou, S., Shi, Y., Wang, C., Li, B., Li. Y., \& Wu, S. (2018). Heavy metals in food crops, soil, and water in the Lithe River Watershed of the Taihu Region and their potential health risks when ingested. Sci. Total Environ., 615, 141-149.

Cioica, N., Tudora, C., luga, D., Deak, G., Matei, M., Nagy, E., \& Gyorgy, Z. (2019). A review on Phytoremediation as an ecological method for in situ clean up of heavy metals contaminated soils. E3S Web of Conferences, 112, 03024

Di Rienzo J. A., Casanoves F., Balzarini M. G., Gonzalez, L., Tablada M., \& Robledo C. W. (2020). InfoStat versión 2020. Centro de Transferencia InfoStat, FCA, Universidad Nacional de Córdoba, Argentina.

Do Carmo, A. S., Rendina, A., Bursztyn, A., De los Ríos, A., Arnedillo, G., \& Lorio, A. (2019). Uso de Lactuca sativa como especie diagnóstico en sedimentos enmendados con compost. V Reunión Argentina de Geoquímica de la Superficie. La Plata.

Duan, J., He, S., Feng, Y., Yu, Y., Xue, L., \& Yang, L. (2017). Floating ryegrass mat for the treatment of low-pollution wastewater. Ecol. Eng., 108, 172-178.

FAO - Food and Agricultural Organization. (2011). Resolución N ${ }^{\circ}$ 12/11 - Reglamento Técnico MERCOSUR sobre límites máximos de contaminantes orgánicos en alimentos.

Gunduz, S., Uygur, F., \& Kahramanoglu, I. (2012). Heavy metal phytoremediation potentials of Lepidum sativum L., Lactuca sativa L., Spinacia oleracea L. and Raphanus sativus L. Herald Journal of Agricultural and Food Science Research, 1(1), 1-5

Habibul, N., Chen, J., Hu, Y., Yin, H., Sheng, G., \& Yu, H. (2019). Uptake, accumulation and metabolization of 1-buty-3methylimidazolium, bromide by ryegrass from water: prospects for phytoremediation. Water Research, 156, 82-91.

NTC. (2011). NTC 5167: Productos para la Industria Agrícola. Productos orgánicos usados como abonos orgánicos 0 fertilizantes y enmiendas del suelo. Instituto Colombiano de Normas Técnicas y Certificaciones: ICONTEC.

Madueño V. F. M. (2017). Determinación de metales pesados (plomo y cadmio) en lechuga (Lactuca sativa) en mercados del Cono Norte, Centro y Cono Sur de Lima Metropolitana. Tesis de pre grado, Universidad Nacional Mayor de San Marcos, Lima. Perú.

Mahar, A., Wang, P., Ali, A., Awasthi, M.K., Lahori, A.H., Wang, Q. \& Zhang, Z. (2016). Challenges and opportunities in the phytoremediation of heavy metals contaminated soils: A review. Ecotoxixol. Environ. Saf., 126, 111-121.
Méndez, P., Sandoval, A., García, P., \& Trejo, N. (2018). Phytoremediation of soils contaminated with heavy metals. Biodiversity International Journal, 2(4), 362-376.

MINAM. (2017). Estándares de Calidad Ambiental (ECA) para Suelo. Decreto Supremo N 011-2017-MINAM. Lima, Perú.

Musa, D., Garba, Y., Yusuf, M., \& Danjuma, M. (2017). Potential of lettuce (Lactuca sativa) for phytoremediation of $\mathrm{Cd}, \mathrm{Cu}, \mathrm{Cr}$ and $\mathrm{Pb}$ in contaminated soil along river Salanta. Dutse Journal of Pure and Applied Sciences, 3(2), 258-269.

Muthusaravanan, S., Sivarajasekar, N., Vivek, J.S., Paramasivan, T., Naushad, M., Prakashmaran, J., Gayathri, V., \& Al-Duaij, O.K. (2018). Phytoremediation of heavy metals: mechanisms, methods and enhancements. Environ. Che. Lett., 16, 1339-1359.

Oconnor, D., Zheng, X., Hou, D., Shen, Z., Li, G., Miao, G., \& Guo, M. (2019). Phytorremediation: climate change resilience and sustainability assessment at a coastal brownfield redevelopment. Environ. Int. 130, 104945.

Proyecto Mantaro Revive. (2007). Avances de resultados de la evaluación de calidad ambiental de los recursos agua y suelo. Data mayo - octubre 2007. Caritas Huancayo.

Purwaningdyah, A., \& Takarina, N. (2019). Translocation factor of Zinc (Zn) in water and sediment by root and stem of Rhizophora sp. at Blanakan Riparian, west Java. Earth and Environmental Science, 550, 102006.

Rajkumar, M., Narasimha, M., Vara, P.F., \& Noriharu, A. (2009). Biotechnological applications of serpentine soil bacteria for phytoremediation of trace metals. Biotechnology, 29(2), 120130.

Ruiz, J. (2012). Evaluación de tratamientos para disminuir cadmio en lechuga (Lactuca sativa L.) regada con agua del río Bogotá. Revista Colombiana de Ciencias Hortícolas., 5(2), 295-302.

SENAMHI (2021). Boletín agroclimático mensual. Servicio Nacional de Meteorología e Hidrología del Perú. Concepción, Junín.

Sharma, S., Singh, B., \& Manchanda, V. K. (2015). Phytoremediation: Role of terrestrial plants and aquatic macrophytes in the remediation of radionuclides and heavy metal contaminated soil and water. Environ. Sci. Pollut. Res., 22(2), 946-962.

Wang, S., Xu, Y., Norbu, N., \& Wang, Z. (2018). Remediation of biochar on heavy metal polluted soils. Earth and Environmental Science 108, 042113.

Yadav, K. K., Gupta, N., Kumar, A., Reece, L. M., Singh, N., Rezania, S., \& Khan, S.A. (2018). Mechanistic understanding and holistic approach of phytoremediation: a review on application and future prospects. Ecol. Eng., 120, 274-298. 